\title{
Bruton's tyrosine kinase: an emerging targeted therapy in myeloid cells within the tumor microenvironment
}

\author{
Logan Good ${ }^{1} \cdot$ Brooke Benner $^{1} \cdot$ William E. Carson ${ }^{2}$ (I)
}

Received: 12 October 2020 / Accepted: 2 March 2021 / Published online: 5 April 2021

(c) The Author(s) 2021

\begin{abstract}
Bruton's tyrosine kinase (BTK) is a non-receptor kinase belonging to the Tec family of kinases. The role of BTK in B cell receptor signaling is well defined and is known to play a key role in the proliferation and survival of malignant B cells. Moreover, BTK has been found to be expressed in cells of the myeloid lineage. BTK has been shown to contribute to a variety of cellular pathways in myeloid cells including signaling in the NLRP3 inflammasome, receptor activation of nuclear factor- $\kappa \beta$ and inflammation, chemokine receptor activation affecting migration, and phagocytosis. Myeloid cells are crucial components of the tumor microenvironment and suppressive myeloid cells contribute to cancer progression, highlighting a potential role for BTK inhibition in the treatment of malignancy. The increased interest in BTK inhibition in cancer has resulted in many preclinical studies that are testing the efficacy of using single-agent BTK inhibitors. Moreover, the ability of tumor cells to develop resistance to single-agent checkpoint inhibitors has resulted in clinical studies utilizing BTK inhibitors in combination with these agents to improve clinical responses. Furthermore, BTK regulates the immune response in microbial and viral infections through B cells and myeloid cells such as monocytes and macrophages. In this review, we describe the role that BTK plays in supporting suppressive myeloid cells, including myeloid-derived suppressor cells (MDSC) and tumor-associated macrophages (TAM), while also discussing the anticancer effects of BTK inhibition and briefly describe the role of BTK signaling and BTK inhibition in microbial and viral infections.
\end{abstract}

Keywords Bruton's tyrosine kinase $\cdot$ BTK $\cdot$ Myeloid-derived suppressor cells $\cdot$ Tumor-associated macrophage

\section{Introduction}

Bruton's tyrosine kinase (BTK) is a non-receptor intracellular kinase that belongs to the Tec (tyrosine kinase expressed in hepatocellular carcinoma) family $[1,2]$. Generally, BTK is located in a cytoplasmic position, yet it can be briefly recruited to the cell membrane via interaction with

Logan Good and Brooke Benner contributed equally to this manuscript.

William E. Carson

william.carson@osumc.edu

Logan Good

logan.good@osumc.edu

1 Comprehensive Cancer Center, The Ohio State University, Columbus, $\mathrm{OH}$, USA

2 Department of Surgery, Division of Surgical Oncology, Tzagournis Medical Research Facility, The Ohio State University, Columbus, OH, USA phosphatidylinositol-3,4,5-triphosphate $\left(\mathrm{PIP}_{3}\right)$, a phospholipid effector activated by phosphatidylinositol-3 kinase (PI3K) [3]. BTK is known for its role in B-cell receptor (BCR) signaling, which is critical for normal B cell development and survival as well as its involvement in the signaling of Toll-like receptors (TLRs), chemokine receptors, and growth factor receptors. However, the expression of BTK is not restricted to B cells [2, 4-8]. Many myeloid cell lineages including monocytes, macrophages, thrombocytes, neutrophils, dendritic cells, and other cell types also express BTK, although the role that BTK plays in myeloid cell function is less defined $[9,10]$. Recently, our group and others have shown that BTK is involved in the function, maturation, and trafficking of myeloid cells and plays an important role in the regulation of myeloid cell signal transduction [9-13]. BTK has an impact on a variety of myeloid cell pathways, including signaling through the NLRP3 inflammasome in neutrophils and macrophages, receptor activation of nuclear factor- $\kappa \beta(\mathrm{NF}-\kappa \mathrm{B}$ in osteoclasts, and the function and activation of myeloid-derived suppressor cells (MDSC) (Fig. 1) 


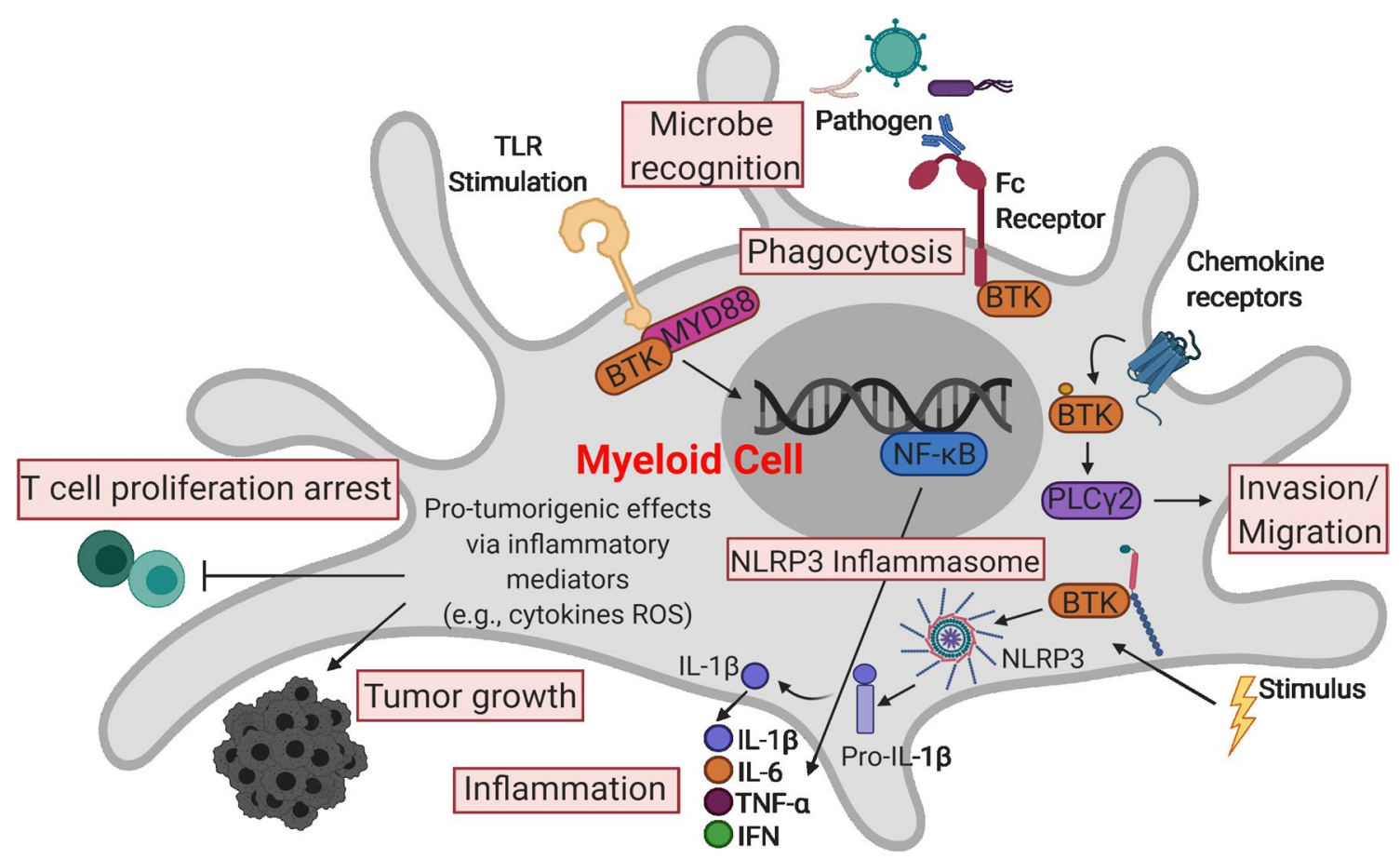

Fig. 1 An overview of the roles of BTK in myeloid cells. The processes highlighted in red boxes indicate roles of BTK signaling in myeloid cells that have been reported in human or mice in the setting of microbial infection and cancer. Details regarding each cellular process are outlined in the text. Abbreviations: BTK, Bruton's tyros- ine kinase; TLR, Toll-like receptors; MYD88, myeloid differentiation primary response 88 ; PLC $\gamma 2$, phospholipase $\mathrm{C}$ gamma 2 ; NF- $\mathrm{\kappa B}$, nuclear factor-kappa B; NO, nitric oxide; IL-1 $\beta$, interleukin 1 beta; IL-6, interleukin 6; TNF- $\alpha$, tumor necrosis factor-alpha; IFN, interferon
[14-18]. Research has shown that BTK also plays a key role in the oncogenic signaling that is critical for the survival and proliferation of several B cell malignancies [19]. BTK has been identified as a therapeutic target in several hematological malignancies, which has driven the development of small-molecule inhibitors that were efficacious in preclinical studies and have gone on to testing and approval in the clinical setting [20, 21]. Finally, it is important to note that BTK is also expressed by T cells and NK cells and is significant in their activation $[22,23]$. However, the focus of this review is on the role of BTK in myeloid cells and the therapeutic implications of targeting BTK in cancer and microbial infections.

\section{BTK in BCR signaling and B cell malignancies and disorders}

BTK is critical for B cell development and BCR signaling. The BCR signaling pathway is important in regulating the ability of B cells to survive, mature, adhere, and produce antibodies as plasma B cells [19, 24-26]. Upon BCR engagement, a signaling cascade involving Lyn, Syk, and BTK results in the activation of phospholipase C $\gamma 2$ (PLC $\gamma 2$ ). In turn, the transcription factor $\mathrm{NF}-\kappa \mathrm{B}$ is activated resulting in the stimulation of survival and proliferative pathways [27-30]. In the absence of BTK, BCR-induced survival and proliferative signals are diminished [27-29].

BTK also contributes to tumor cell proliferation and survival in B cell leukemia and lymphoma [31]. Evidence suggests that overactive signaling supports the development of the tumor microenvironment through aberrant chemokinecontrolled integrin-mediated adhesion and migration of malignant cells [26, 32]. Various small molecule inhibitors have been developed to target protein kinases in the BCR signaling cascade, and these have shown promising clinical effectiveness in multiple disease settings [2, 33]. Overall, this emphasizes the potential of targeting molecules associated with the BCR signaling pathway.

Defects in BTK have been implicated in abnormal B cell function. One major consequence of BTK impairment is the developmental delay of pro-B cells to pre-B cells and onto mature B lymphocytes. In 1993, BTK was identified as the gene defective in X-linked agammaglobulinemia (XLA), an inherited immunodeficiency disease in humans characterized by a drastic deficiency in B lymphocytes, low levels of immunoglobulin (Ig), and recurring infections [34-37]. Mutations in the pleckstrin homology $(\mathrm{PH})$ domain reduce the binding activity of BTK and eliminate downstream signaling, which was identified in the murine version of BTK 
deficiency known as X-linked immunodeficient (XID). XID presents with a set of physiological manifestations similar to that of XLA in humans, yet is not as severe [34-38]. Both XLA in humans and XID in mice result in the depletion of B cells.

\section{BTK in immune regulation and function}

BTK is also expressed by other immune cell populations and has been implicated in the immune regulation and function of myeloid cells. BTK has been shown to play an important role in many TLR signaling pathways. TLRs are both extracellular and intracellular pattern recognition receptors that are expressed in B cells and myeloid cells. They recognize structurally conserved molecules from bacteria and viruses [19]. Upon activation by pathogens, most TLRs recruit the adaptor myeloid differentiation primary response 88 (MyD88) which activates interleukin-1 receptor-associated kinase1 (IRAK1). This results in the activation, proliferation, antibody secretion, class switch recombination, and pro-inflammatory cytokine production in B cells [19, 39-41]. Studies investigating non-canonical pathways activated downstream of TLRs have shown that BTK can interact with the adapter molecules MyD88 and MAL downstream of TLR4, 6, 8, and 9 ultimately resulting in the production of IL-6 and TNF- $\alpha$ (Fig. 1) [42, 43]. Additionally, BTK is required for TLR-mediated IL-10 production in $\mathrm{B}$ cells and the cooperation between BCR and TLR signaling in the enhancement of IL-6 expression [38]. Kawakami et al. reported that BTK is required for IL-10 secretion by DCs and subsequent activation of STAT3 [13]. Notably, in the setting of BTK deficiency, dendritic cell cytokine production is impaired in response to viral single-stranded RNAs that activate TLR- 8 -mediated TNF- $\alpha$ and IL- 6 production (Fig. 1) [6].

Furthermore, BTK is a contributor to chemokine receptor signaling pathways. Integrin $\alpha 4 \beta$ (VLA-4)-mediated adhesion of B cells to vascular cell adhesion molecule-1 and fibronectin is dependent on BTK, as are B cell responses to various chemokines like stromal cell-derived factor 1 (SDF1) and chemokine (C-X-C motif) ligand 13 (CXCL-13) [44-46]. Chemokine receptors are G-protein-coupled receptors that are composed of seven transmembrane regions, an extracellular $\mathrm{N}$-terminus contributing to ligand specificity, and intracellular hetero-trimeric $\mathrm{G}$ proteins comprised of $\alpha, \beta$, and $\gamma$ subunits $(\mathrm{G} \alpha, \mathrm{G} \beta$, and $\mathrm{G} \gamma[47,48]$. After a chemokine binds to the extracellular domain of the receptor, downstream activation of PI3K leads to the activation of BTK (Fig. 1) [49]. Furthermore, the G $\alpha$ and G $\beta \gamma$ subunits can both interact with the $\mathrm{PH}$ and the Tec homology (TH) domain to directly bind to BTK $[50,51]$. However, the $\mathrm{G} \alpha$ subunit has been shown to directly activate BTK alone
[52]. B cells express chemokine receptors such as CXCR4 and CXCR5, and BTK is critical in acting downstream to mediate chemokine-controlled migration and maintain cell homeostasis [45].

The $\mathrm{Fc}$ receptor is an antibody receptor expressed by a multitude of cells including B cells, macrophages, mast cells, natural killer (NK) cells, and neutrophils. These receptors recognize the constant region of immunoglobulin G, known as the $\mathrm{Fc}$ (fragment, crystallizable) region. Among others, the Fc epsilon receptor (FCERI)-mediated crosslinking in mast cells strongly activates BTK, which plays a role in many $\mathrm{Fc}$ receptors [12, 52, 53]. An activating $\mathrm{Fc}$ receptor (FceRI) and immunoreceptor tyrosine-based activation motifs (ITAMs) recruit downstream messengers to activate various kinases such as SYK and BTK upon antigen recognition and cross-linking, but if an inhibitory Fc receptor such as Fc $\gamma$ RIIB recognizes an antigen, immunoreceptor tyrosine-based inhibitory motifs (ITIMs) recruit phosphatases to inhibit the effector function of BTK and eliminate downstream signaling (Fig. 1) [54]. Importantly, Bolland et al. reported that membrane recruitment of SHIP is responsible for the inhibitory signal generated by Fc $\gamma$ RIIB to the BCR [55].

There is evidence that BTK can play a role in the cellular process of phagocytosis (Fig. 1). It was reported in a murine macrophage cell line RAW 264.7 that BTK was activated throughout phagocytosis and inhibition of BTK resulted in significantly inhibited Fc $\gamma$ R-mediated phagocytosis [56]. BTK also contributes to phagocytosis in rodent microglia and human monocyte-derived macrophages as well as in cells from patients with XLA, a condition caused by a BTK mutation [57, 58]. However, further studies are needed to determine whether these effects are a result of a direct effect of the BTK inhibition.

\section{BTK inhibition}

Due to its role in B cell development, the BCR signaling cascade, and other immune-related pathways, BTK serves as a unique therapeutic target for many B cell malignancies and other disease states. Multiple agents have been developed for BTK inhibition, [33, 59] but this review will focus on the BTK inhibitors ibrutinib and acalabrutinib which have been tested extensively in the clinical realm.

Ibrutinib or PCI-32765 (brand name Imbruvica) is an orally available, irreversible inhibitor of BTK that binds to the cysteine at position 481 in the kinase domain where it blocks BTK kinase activity [60, 61]. Ibrutinib has been evaluated in several preclinical studies since its initial discovery in 2007. Ibrutinib inhibits the autophosphorylation of BTK, the phosphorylation of its substrate PLC $\gamma 2$, and the downstream extracellular-signal-regulated kinase (ERK), leading 
to reduced activation of NF- $\mathrm{KB}$ signal transduction $[33,62]$. Furthermore, ibrutinib has a potent (inhibitory concentration $\left.50\left(\mathrm{IC}_{50}\right)=0.5 \mathrm{nM}\right)$ selectivity for BTK in B cells as opposed to other Tec kinases, making it an attractive therapeutic approach for B cell malignancies [33]. An important in vivo study of ibrutinib by Honiberg et al. reported objective clinical responses in dogs with spontaneous B cell nonHodgkin lymphoma [55]. Furthermore, the report indicated that ibrutinib-targeted BTK yet displayed off-target effects on other kinases with a corresponding cysteine residue in the ATP-binding site including JAK and ITK. These off-target effects of ibrutinib could explain unique toxicities and why ibrutinib has been found useful in other diseases [63-68]. Clinical efficacy was first reported in patients with chronic lymphocytic leukemia (CLL) and mantle cell lymphoma (MCL) [69]. Ibrutinib received breakthrough designation and was later approved by the Food and Drug Administration (FDA) for treatment of MCL in 2013 and CLL in 2014 $[20,21]$.

Acalabrutinib or ACP-196 (brand name Calquence) is an orally available, more selective second-generation irreversible BTK inhibitor that was designed to improve the safety and efficacy of first-generation inhibitors like ibrutinib [70]. Acalabrutinib binds irreversibly to cysteine 481 in the BTK kinase domain and blocks kinase activity, but results in less off-target activity on kinases like EGFR and is predicted to have fewer adverse events (AEs) than ibrutinib, such as antiplatelet activity [70, 71]. Acalabrutinib has shown success in clinical trials, including the first clinical use of the BTK inhibitor for patients with relapsed CLL such as those with chromosome 17p13.1 deletion. In this clinical setting, a 95\% overall response rate was reported [72]. Furthermore, a phase II trial in refractory MCL patients reported a complete response in $40 \%$ of patients [73]. Acalabrutinib became FDA approved for adult patients with MCL in 2017 and CLL in 2019. An ongoing phase III clinical study investigating the relative effectiveness of acalabrutinib and ibrutinib in previously treated subjects with CLL (NCT02477696) has pending results [74].

\section{The role of BTK in myeloid-derived suppressor cells (MDSC)}

Myeloid-derived suppressor cells (MDSC) are a population of heterogeneous myeloid progenitor cells that can inhibit $\mathrm{T}$ cell function and have been identified as contributors to the progression of many different types of cancer $[75,76]$. MDSC are known to expand and activate as the result of two distinct signals. The first signal functions to expand immature myeloid cell populations, while the second signal is an activation signal, primarily mediated by the transcription factor NF- $\mathrm{KB}$ [77]. After activation, MDSC are responsible for producing various anti-inflammatory cytokines, nitric oxide, reactive oxygen species (ROS), and arginase-1, all of which function to suppress immune function $[75,76]$. BTK plays an important role in the maturation and function of myeloid cells, and targeting BTK in malignant B cells has been shown to inhibit NF-KB signal transduction [14, $15,17,62]$. Notably, NF- $\mathrm{\kappa B}$ is a demonstrated mediator of MDSC expansion and function [78, 79]. MDSC expansion can cause loss of immune effector cell function and reduce the efficacy of immune-based cancer treatments, highlighting the therapeutic benefit of potentially targeting BTK in the MDSC population [17].

Stiff et al. found that MDSC isolated from Balb/c mice bearing EMT6 and 4T1 mammary carcinoma tumors, human MDSC generated in vitro, and MDSC isolated from metastatic melanoma patients all expressed BTK [17]. Furthermore, treatment of human MDSC and the murine MDSC cell line MSC2 with the BTK inhibitor ibrutinib led to inhibition of BTK phosphorylation in these cells [17]. Notably, ibrutinib also significantly inhibited the in vitro generation of MDSC, impaired MDSC migration, reduced mRNA expression of the immunosuppressive factor indolamine 2,3-dioxygenase, and halted production of the immunosuppressive molecule nitric oxide (NO) [17]. Our group has previously shown that cancer patient MDSC produce $\mathrm{NO}$ which significantly inhibits NK cell Fc receptor-mediated functions, and eliminating MDSC in vivo significantly improved monoclonal antibody treatment in an EMT6-HER2 mammary carcinoma model [80]. Ibrutinib treatment in wild-type mice bearing $\mathrm{B} 16 \mathrm{~F} 10$ melanoma tumors also resulted in a significant reduction of MDSC [17]. In Balb/c mice bearing EMT6 murine mammary carcinoma tumors, treatment with ibrutinib resulted in a significant reduction of MDSC in both the spleen and tumor and was shown to significantly enhance anti-PD-L1 therapy [17]. Taken together, these results demonstrated that targeting BTK with ibrutinib can modulate MDSC function, generation, and migration, revealing a promising strategy for enhancing immune modulation for solid tumor therapies.

The ability of ibrutinib to modulate MDSC has also been demonstrated utilizing an orthotopic mouse breast cancer model [81]. Varikuti et al. reported that ibrutinib treatment inhibited the proliferation of the E0.2 breast cancer cell line in vitro, while additionally demonstrating through a proliferation assay with CFSE-treated cells (carboxyfluorescein succinimidyl ester) that T cells co-cultured with MDSC from the spleens of ibrutinib-treated mice can proliferate significantly more compared to controls while promoting effector functions leading to the induction of anti-tumor $\mathrm{T}$ helper type 1 (Th1) and cytotoxic T lymphocytes (CTL) immune responses [17, 81]. Additionally, the spleens and tumors of ibrutinib-treated mice had significantly reduced levels of monocytic MDSC (M-MDSC), but the levels of granulocytic 
MDSC (PMN-MDSC) exhibited no significant change [81]. High levels of M-MDSC have been correlated with breast cancer metastasis and progression in human breast cancer patients [82]. Thus, it was not surprising for this group to find that ibrutinib-treated mice had a significant reduction in tumor metastasis to the lungs, a lower tumor burden, and reduced breast tumor progression compared to controls [81]. Treatment with ibrutinib also increased the frequency of mature dendritic cells (DCs) in the spleens and tumors of mice by converting MDSC to mature DCs, which are known to play a critical role in anti-tumor immunity [81-83]. These findings were confirmed via an ex vivo treatment of MDSC with ibrutinib which significantly enhanced expression of CD11c and MHCII molecules, indicating a change in phenotype to mature DCs [81]. These findings suggest the BTK pathway negatively regulates the conversion of MDSC into mature DCs [81]. Overall, these results demonstrate that ibrutinib inhibits tumor development and metastasis in an E0.2 breast cancer mouse model by inducing the transformation of mature DCs from MDSC, providing a novel, promising approach for the treatment of human breast cancer [81].

It is hypothesized that MDSC could limit the effectiveness of checkpoint inhibitors and that BTK inhibition could improve the effectiveness of checkpoint inhibitors by eliminating the negative effects of MDSC. As a result, combination clinical studies are being performed with BTK and checkpoint inhibition in patients with solid malignancies (Table 1). Overman et al. recently conducted a phase II, multicenter, open-label, randomized (1:1) clinical trial (NCT02362048) evaluating the BTK inhibitor acalabrutinib alone or in combination with the anti-PD-1 antibody pembrolizumab in patients with advanced pancreatic cancer [84]. Overall, 77 patients (37 monotherapy, 40 combination therapy) with a median age of 64 years received $100 \mathrm{mg}$ of oral acalabrutinib twice daily with (combination therapy) or without (monotherapy) $200 \mathrm{mg}$ of intravenous pembrolizumab on day 1 of each 3 -week cycle, as peripheral blood and some tumors were analyzed [84]. The elevation of peripheral MDSC has been correlated with disease progression in patients with solid tumors treated with anti-cytotoxic T-lymphocyte-associated protein-4 (antiCTLA-4), anti-PD-1, or anti-PD-L1 therapies [85, 86]. Flow cytometric analysis of peripheral blood mononuclear cells (PBMC) demonstrated durable reductions in the levels of PMN-MDSC, with a median reduction of greater than 50\% achieved after 2-3 weeks of therapy in both arms of the study, suggesting this effect was likely facilitated by the BTK inhibitor acalabrutinib [84]. Furthermore, two exceptional responders in the study were shown to have low tumor mutational burden and no defects in the homologous DNA repair pathway [84]. Overall, the Overman et al. study showed that combination therapy with ibrutinib and pembrolizumab was well tolerated in patients with advanced pancreatic cancer, but limited clinical benefit was achieved in both arms of the study [84]. However, the study did demonstrate the strong effect on eliminating peripheral PMN-MDSC levels, emphasizing the importance of further studying the role of MDSC in the tumor microenvironment. Furthermore, a phase I clinical trial (NCT03525925), studying the effect of ibrutinib and anti-PD-1 inhibitor nivolumab on the levels of circulating MDSC in patients with metastatic solid tumors, is ongoing at our institution. Early results from this study show an initial increase in circulating MDSC levels with single-agent ibrutinib followed by reductions with the combination regimen. Plasma levels of chemokines (IL-8, CCL2, CCL3, and CCL4) associated with MDSC recruitment and migration significantly decreased following ibrutinib treatment and $\mathrm{T}$ cell function significantly improved with the combination therapy. Taken together, these results demonstrate the ability of ibrutinib to modulate MDSC in the setting of cancer and provide biological evidence to support the expansion of strategies to target MDSC in combination with immune checkpoint inhibition [87]. Overall, BTK inhibition has shown a favorable benefit in solid tumors with anti-tumor activity; however, these data are still preliminary, and it is possible that these effects are a result of the off-target effects of BTK inhibitors on other kinases.

\section{The role of BTK in tumor-associated macrophages (TAM)}

In cancer, macrophages can be recruited to the tumor microenvironment, and their function modified by tumor-derived factors. These tumor-resident macrophages may be roughly characterized as tumor-associated macrophages (TAM) [88]. Importantly, TAM can promote tumor growth and metastasis, enhance cancer cell resistance to chemotherapy, activate immunosuppression pathways, and inactivate $\mathrm{T}$ cell effector functions $[88,89]$. Sousa et al. demonstrated in a study of human breast cancer that higher levels of TAM were correlated with increased tumor cell proliferation, augmented tumor growth, significant immunosuppression, and a subsequent increase in disease burden [90]. TAM are one of the most abundant tumor-infiltrating immune cells in the tumor microenvironment and play an important role in suppressing the anticancer immune response. Thus, TAM have become an attractive therapeutic target [91].

One mechanism by which TAM protect cancer cells and promote pro-survival signaling is through the secretion of chemokines such as CXCL12 and CXCL13, which bind to the G-protein-coupled receptors CXCR4 and CXCR5, respectively, found on malignant cells [92]. Due to the role that BTK plays in macrophage production of many proinflammatory chemokines and cytokines, Ping et al. investigated the immunomodulatory effects of BTK inhibition 


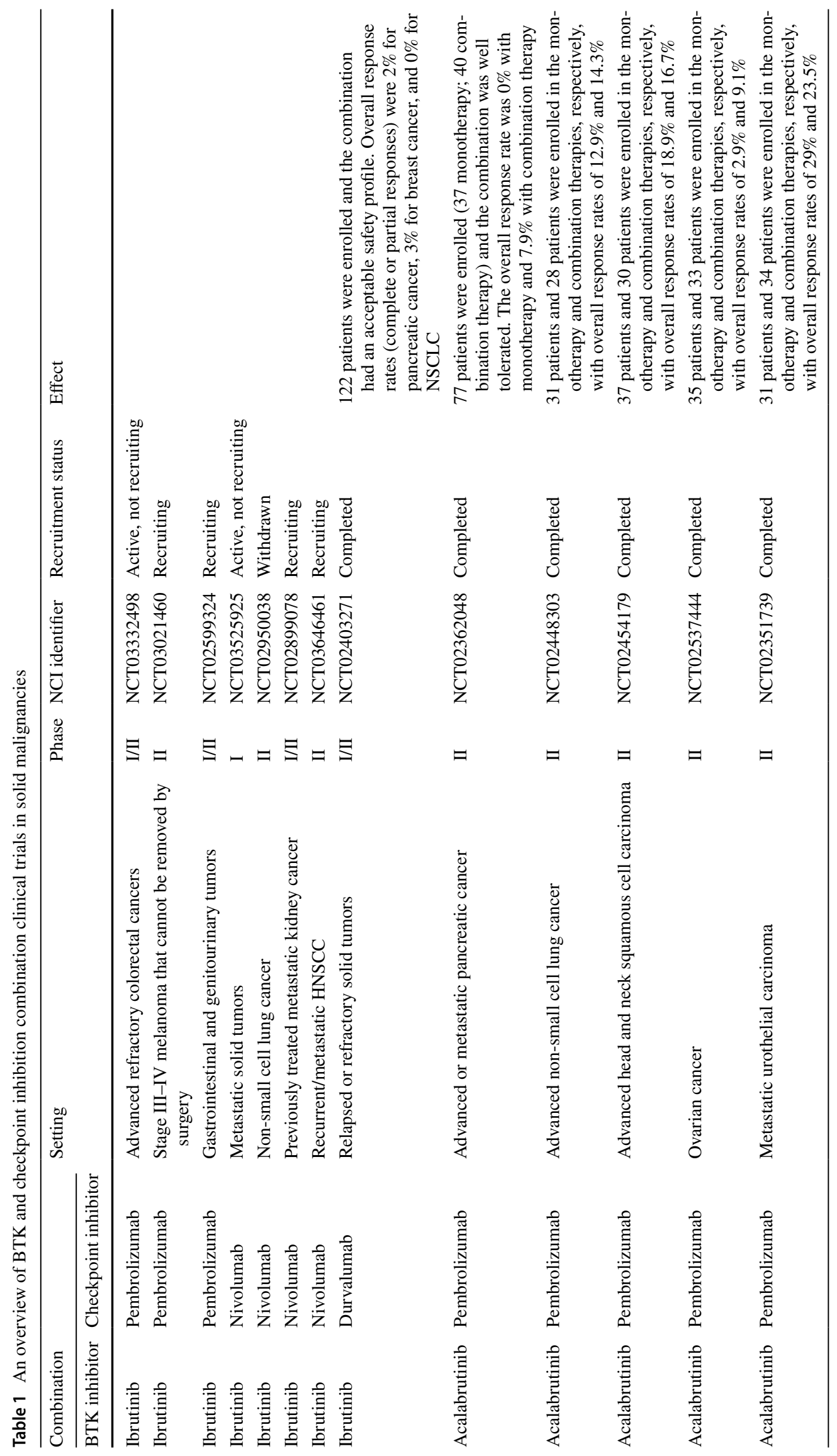


on macrophages [92]. This group discovered that BTK inhibition with ibrutinib or with PLS-123 (targets BTK catalytic activity and its auto-activation) in LPS-stimulated THP-1 differentiated macrophages efficiently downregulated the secretion of the homeostatic chemokines CXCL12, CXCL13, and CCL19, as well as the angiogenic cytokine VEGF [92]. Inhibition of these factors also occurred at the mRNA level, demonstrating the role of BTK in regulating the transcription of these genes [92]. In addition to targeting BTK pharmacologically, Ping et al. performed siRNA-mediated knockdown of BTK which also significantly impaired the secretion of CXCL12, CXCL13, CCL19, and VEGF [92]. Moreover, macrophages co-cultured with malignant B cell lymphoma cells (Namalwa or OCI-Ly7 cell lines) exhibited diminished adhesion to fibronectin compared to control [92]. A transwell migration assay demonstrated that malignant $\mathrm{B}$ cell and $\mathrm{T}$ cell lymphoma cells had diminished ability to migrate and invade when cultured with the supernatants of ibrutinib-treated cells [92]. Further experiments in macrophages revealed that BTK inhibitors impede downstream signaling pathways such as PLC $\gamma 2$ and MAP kinases and the activity of specific transcription factors important for controlling chemokine and cytokine production such as NF- $\mathrm{BB}$, STAT3, and AP-1 [92]. These results suggest that BTK inhibitors can be used to target TAM in the tumor microenvironment and modify the immune landscape via modulation of the chemokine and cytokine milieu.

Others have studied the role of TAM in murine models of cancer, particularly in pancreatic ductal adenocarcinoma (PDAC)-bearing mice [93]. One characteristic feature of PDAC is the infiltration of various lymphoid and myeloid cell lineages, like TAM, which exert a strong effect on the tumor microenvironment and often contribute toward the ineffectiveness of various therapies [94]. Gunderson et al. investigated the role of the BTK signaling pathway based on data indicating that B cells and TAM can contribute to PDAC disease progression and tumorigenesis by inhibiting cytotoxic $\mathrm{T}$ lymphocyte (CTL) responses. This group found that PDAC tumor growth requires crosstalk between $\mathrm{B}$ cells and TAM, resulting in pro-tumor Th2-type macrophage polarization via activation of the BTK signaling cascade [93]. In fact, one of the main features of an immunosuppressive environment was the lack of functional CTL due to macrophage polarization into a Th2-type phenotype [2]. Treatment of PDAC-bearing mice with ibrutinib reprogrammed macrophages toward a Th1-type macrophage phenotype which led to the enhancement of $\mathrm{CD} 8^{+} \mathrm{CTL}$ responses, improvement of a chemotherapeutic intervention with gemcitabine, and suppression of PDAC tumor progression. Thus, BTK appears to serve as a key regulator of TAM crosstalk and anti-tumor immune responses [93]. Unfortunately, in some cases in vivo ibrutinib is rapidly cleared, and thus, the low accumulation of ibrutinib results in the tumor, rendering these targeted therapies ineffective [95]. Qiu et al. have designed, synthesized, and investigated a sialic acid-stearic acid (SA) conjugate that encapsulates the BTK inhibitor ibrutinib within amphiphilic egg phosphatidylglycerol (EPG) as a novel immunotherapeutic approach to allow for prolonged exposure of TAM to ibrutinib [95]. Immunofluorescence staining demonstrated that the SA/IBR/EPG nanocomplex accumulated in TAM both in vitro and in vivo. This agent inhibited angiogenesis, Th2 tumorigenic cytokine secretion, and tumor progression and represents a promising targeted approach for targeting BTK in TAM [95].

Benner et al. have also studied the role that the BTK signaling pathway may have in modulating TAM-produced inflammatory processes [15]. The NLRP3 inflammasome is a multi-protein complex involved in innate immunity. It is composed of NLRP3, the adaptor protein ASC, and caspase- 1 and is responsible for the production of the potent inflammatory cytokine IL-1 $\beta$ [96]. Due to the inherent role of BTK in myeloid cell signal transduction, it was hypothesized that in vitro generated TAM would express BTK and that this enzyme would participate in NLRP3 inflammasome activity $[15,97]$. Murine and human TAM (both in vitro generated and tumor-derived) were utilized to establish that TAM express both BTK and the NLRP3 inflammasome [15]. Moreover, it was demonstrated that BTK physically associates with the NLRP3 inflammasome and promotes its activation and subsequent ability to mediate the production of IL-1 $\beta$ [15]. IL- $1 \beta$ production could be induced in TAM using LPS and ATP co-stimulation and ibrutinib inhibited the phosphorylation of BTK, prevented the association of BTK with the NLRP3 inflammasome, and reduced inflammasome activation as evidenced by a decrease in IL- $1 \beta$ production [15]. Furthermore, compared to controls, systemic ibrutinib therapy led to reduced expression of IL- $1 \beta$ expression in murine EMT6 and 4T1 tumors. These results suggest that BTK plays an important role in TAM-mediated inflammation within the tumor microenvironment that involves around IL-1 $\beta[15]$.

Other groups have studied the association between BTK and the NLRP3 inflammasome in different disease settings. Liu et al. used proteome-wide phosphoproteomics to identify and study BTK as a novel regulator of the NLRP3 inflammasome [18]. Utilizing bone marrow-derived macrophages from BTK-deficient mice and PBMC from patients with $\mathrm{X}$-linked agammaglobulinemia (XLA), this group found reduced IL- $1 \beta$ production and impaired inflammasome activation within mutant myeloid cells [18]. Also, BTK inhibition impaired IL- $1 \beta$ processing and release in human primary macrophages from healthy donors, ibrutinib-treated cancer patients, and patients with Muckle-Wells syndrome-an autoinflammatory disease characterized by excessive production of IL-1 $\beta$ [18]. Inflammasome activation has also been implicated in the post-ischemic inflammation that 
occurs after stroke $[98,99]$. Using a murine model of brain ischemia/reperfusion, Ito et al. reported that BTK inhibition by genetic and pharmacologic means impaired inflammasome activation in brain infarct macrophages and exerted a neuroprotective effect [14]. Immunofluorescence staining demonstrated that BTK inhibition suppressed caspase-1 activation in the brain infarct and correlated with the reduced maturation of IL-1 $\beta$ [14]. These studies offer further evidence for BTK as a crucial mediator of myeloid cell function and NLRP3 inflammasome activation and point toward the potential for BTK inhibition as a means of improving clinical outcomes in cancer and other diseases.

\section{Targeting BTK beyond the tumor microenvironment}

Myeloid cell lineages contribute to inflammation and infection, making BTK an interesting candidate for therapeutic intervention in a variety of disease settings [100]. TLR signaling plays an important role in innate immunity and TLRs are expressed in many myeloid cell populations including dendritic cells, mast cells, and macrophages, which are all essential for the recognition and elimination of microbial pathogens [101]. BTK promotes TLR signaling, leading to the activation of pro-inflammatory cytokines resulting in robust immune responses in many bacterial infections like pneumococcal pneumonia [102]. Murine models have demonstrated the protection BTK confers from endotoxin shock caused by the Gram-negative bacteria Escherichia coli [40]. Furthermore, BTK regulates macrophage responses to the Gram-positive bacteria Listeria monocytogenes and Staphylococcus aureus infections [103, 104]. In filarial infections, Mukhopadhyay et al. revealed that BTK-mutant CBA/N mice showed delayed clearance of microfilaria compared to wild-type CBA/N mice and that BTK regulates macrophage effector functions such as bactericidal activity and secretion of pro-inflammatory cytokines $[10,105]$. However, the BTK inhibitor ibrutinib has been shown to impair immune mechanisms dedicated to controlling the Mycobacterium tuberculosis infection [106].

BTK is also involved in the clearance of fungal infections. Specifically, BTK contributes to the dectin-1-dependent phagocytosis of Candida albicans by macrophages [107]. Furthermore, Shahan et al. demonstrated that BTK is activated in the presence of spores from the fungal species Aspergillus niger, Aspergillus candidus, and Eurotium amstelodami [108]. BTK-deficient XID mice had enhanced susceptibility to Cryptococcus neoformans infection, a fungal pathogen that can affect immunocompromised patients [109]. In other studies, inhibition of the BTK signaling pathway with ibrutinib led to reduced NF- $\mathrm{kB}$ signaling and resulted in an increased risk of developing Aspergillus fumigatus, an invasive fungus largely found in organ transplant recipients $[110,111]$. Recently, others have also demonstrated that second-generation BTK inhibitors impair the antifungal response of macrophages [112].

Various inflammatory diseases are implicated with the BTK signaling pathway. BTK regulates osteoclast differentiation by linking receptor activator of nuclear factor- $\mathrm{\kappa B}$ (RANK) and immunoreceptor tyrosine-based activation motif (ITAM) signals, and inhibiting BTK protects against osteoclast-mediated bone loss disorders $[16,113]$. Other groups have shown that BTK is needed to drive macrophage activation and could be a potential target for patients with rheumatoid arthritis and sepsis [114, 115]. BTK inhibition ameliorates autoimmune arthritis and treatsTLR7/IFNdriven murine lupus, while BTK-deficient XID mice show reduced severity in many inflammatory disease models such as autoimmune encephalomyelitis (EAE), colitis, and acute edema [97, 116, 117]. Finally, Mao et al. demonstrated that BTK regulates NLRP3 inflammasome activity in the setting of Crohn's disease [118].

In addition to inflammatory diseases, bacterial and fungal infections, BTK plays a critical role in initiating antiviral responses [119]. In some pulmonary viral infections, the macrophage response driven by BTK can lead to tissue damage and unfavorable outcomes. For example, in murine models of influenza viral infection BTK inhibition with ibrutinib decreased lung injury and led to reduced alveolar macrophage activation [120]. Recently, Chong et al. and others discussed the potential that BTK inhibition may have in treating the novel virus SARS-CoV-2 (COVID-19) [121-123]. Pulmonary failure is the main cause of mortality related to COVID-19 infection, and BTK inhibition may mitigate the hyper-inflammatory pulmonary response through attenuation of M1-macrophage polarization [121, 124, 125]. Our group presented that the BTK inhibitor ibrutinib could inhibit inflammasome activation in TAMs and reduce the release of IL-1 $\beta$ (see below) [15]. These findings suggest a potential mechanism by which ibrutinib can inhibit macrophage activation, lower the production of IL-1 $\beta$, and alter the pulmonary inflammatory landscape in patients infected with COVID-19. Furthermore, one clinical study utilized the BTK inhibitor acalabrutinib in COVID-19 patients. Administration of acalabrutinib led to improved oxygenation in a majority of patients over the 10-14-day treatment course and was well tolerated. Measures of inflammation such as C-reactive protein and IL-6 rapidly returned to normal in most patients, as did lymphopenia [122]. These findings support the hypothesis that the release of pro-inflammatory cytokines by pulmonary macrophages in COVID-19 is a major contributor to pulmonary failure and that BTK inhibition could provide some degree of protection against lung injury in this setting. BTK plays a large role in macrophage polarization, and through a variety of transcription factors, it 
may effectively regulate the hyperinflammatory state associated with many microbial infections and inflammatory disease settings.

\section{Conclusion}

Much progress has been made in understanding the mechanism by which BTK regulates innate immunity. Targeting BTK in CLL could have significant clinical efficacy via inhibition of the BCR signaling pathway in addition to the significant effects of BTK inhibition on B cell adhesion and chemotaxis. Moreover, targeting BTK in myeloid cells has shown recent progress and holds therapeutic promise for modulating the tumor microenvironment. However, more work is needed to determine the clinical efficacy of BTK inhibition in various disease states. One avenue of particular interest is utilizing BTK inhibition in combination with other immune-modulating agents to combat resistance to single-agent therapies. A greater understanding of the differences in safety profiles and clinical benefit of available BTK inhibitors in different disease states is needed in order to design effective treatment strategies.

Acknowledgments BioRender was utilized for figure development.

Authors' contributions LG, BB, and WEC conceptualized and designed the manuscript. LG and BB wrote the first draft and WEC provided an editorial review.

Funding This work was supported by National Institutes of Health Grants P01CA95426, K24 CA93670 (W.E. Carson), and T32CA9033827 (W.E. Carson).

Open Access This article is licensed under a Creative Commons Attribution 4.0 International License, which permits use, sharing, adaptation, distribution and reproduction in any medium or format, as long as you give appropriate credit to the original author(s) and the source, provide a link to the Creative Commons licence, and indicate if changes were made. The images or other third party material in this article are included in the article's Creative Commons licence, unless indicated otherwise in a credit line to the material. If material is not included in the article's Creative Commons licence and your intended use is not permitted by statutory regulation or exceeds the permitted use, you will need to obtain permission directly from the copyright holder. To view a copy of this licence, visit http://creativecommons.org/licenses/by/4.0/.

\section{References}

1. Hussain A, Yu L, Faryal R et al (2011) TEC family kinases in health and disease-loss-of-function of BTK and ITK and the gain-of-function fusions ITK-SYK and BTK-SYK. FEBS J 278:2001-2010. https://doi.org/10.1111/j.1742-4658.2011. 08134.x
2. Molina-Cerrillo J, Alonso-Gordoa T, Gajate P, Grande E (2017) Bruton's tyrosine kinase (BTK) as a promising target in solid tumors. Cancer Treat Rev

3. Hendriks RW, Yuvaraj S, Kil LP (2014) Targeting Bruton's tyrosine kinase in B cell malignancies. Nat Rev Cancer 14:219-232. https://doi.org/10.1038/nrc3702

4. Genevier HC, Hinshelwood S, Gaspar HB et al (1994) Expression of Bruton's tyrosine kinase protein within the B cell lineage. Eur J Immunol 24:3100-3105. https://doi.org/10.1002/eji.18302 41228

5. Marron TU (2009) The role of BTK in TLR signaling. J Allergy Clin Immunol 123:S92-S92. https://doi.org/10.1016/j.jaci.2008. 12.329

6. Levy O (2007) Bruton tyrosine kinase (Btk): key for signaling via Toll-like receptor 8. Blood 109:2273-2274. https://doi.org/ 10.1182/blood-2006-12-064642

7. Seda V, Mraz M (2015) B-cell receptor signalling and its crosstalk with other pathways in normal and malignant cells. Eur J Haematol 94:193-205. https://doi.org/10.1111/ejh.12427

8. Smith CI, Baskin B, Humire-Greiff P et al (1994) Expression of Bruton's agammaglobulinemia tyrosine kinase gene, BTK, is selectively down-regulated in $\mathrm{T}$ lymphocytes and plasma cells. $\mathrm{J}$ Immunol 152:557-565

9. Horwood NJ, Mahon T, McDaid JP et al (2003) Bruton's tyrosine kinase is required for lipopolysaccharide-induced tumor necrosis factor $\alpha$ production. J Exp Med 197:1603-1611. https://doi.org/ 10.1084/jem.20021845

10. Mukhopadhyay S, Mohanty M, Mangla A et al (2002) Macrophage effector functions controlled by Bruton's Tyrosine kinase are more crucial than the cytokine balance of $\mathrm{T}$ cell responses for microfilarial clearance. J Immunol 168:2914-2921. https://doi. org/10.4049/jimmunol.168.6.2914

11. Sochorová K, Horváth R, Rožková D et al (2007) Impaired Toll-like receptor 8-mediated IL- 6 and TNF- $\alpha$ production in antigen-presenting cells from patients with X-linked agammaglobulinemia. Blood 109:2553-2556. https://doi.org/10.1182/ blood-2006-07-037960

12. Kawakami Y, Yao L, Miura T et al (1994) Tyrosine phosphorylation and activation of Bruton tyrosine kinase upon Fc epsilon RI cross-linking. Mol Cell Biol 14:5108-5113. https://doi.org/10. 1128/MCB.14.8.5108

13. Kawakami Y, Inagaki N, Salek-Ardakani S et al (2006) Regulation of dendritic cell maturation and function by Bruton's tyrosine kinase via IL-10 and Stat3. Proc Natl Acad Sci 103:153-158. https://doi.org/10.1073/pnas.0509784103

14. Ito M, Shichita T, Okada M et al (2015) Bruton's tyrosine kinase is essential for NLRP3 inflammasome activation and contributes to ischaemic brain injury. Nat Commun 6:7360. https://doi.org/ 10.1038/ncomms8360

15. Benner B, Scarberry L, Stiff A et al (2019) Evidence for interaction of the NLRP3 inflammasome and Bruton's tyrosine kinase in tumor-associated macrophages: implications for myeloid cell production of interleukin-1beta. Oncoimmunology 1659704. https://doi.org/10.1080/2162402X.2019.1659704

16. Shinohara M, Koga T, Okamoto K et al (2008) Tyrosine kinases Btk and Tec regulate osteoclast differentiation by linking RANK and ITAM signals. Cell 132:794-806. https://doi.org/10.1016/j. cell.2007.12.037

17. Stiff A, Trikha P, Wesolowski R et al (2016) Myeloid-derived suppressor cells express Bruton's Tyrosine Kinase and can be depleted in tumor-bearing hosts by Ibrutinib treatment. Cancer Res 76:2125-2136. https://doi.org/10.1158/0008-5472. CAN-15-1490

18. Liu X, Pichulik T, Wolz O-O et al (2017) Human NACHT, LRR, and PYD domain-containing protein 3 (NLRP3) inflammasome activity is regulated by and potentially targetable through Bruton 
tyrosine kinase. J Allergy Clin Immunol 140:1054-1067.e10. https://doi.org/10.1016/j.jaci.2017.01.017

19. Pal Singh S, Dammeijer F, Hendriks RW (2018) Role of Bruton's tyrosine kinase in B cells and malignancies. Mol Cancer 17:57. https://doi.org/10.1186/s12943-018-0779-z

20. Byrd JC, Brown JR, O'Brien S et al (2014) Ibrutinib versus Ofatumumab in previously treated chronic lymphoid leukemia. $\mathrm{N}$ Engl J Med 371:213-223. https://doi.org/10.1056/NEJMoa1400 376

21. Wang Y, Eldridge N, Metersky ML et al (2014) National trends in patient safety for four common conditions, 2005-2011. N Engl J Med 370:341-351. https://doi.org/10.1056/NEJMsa1300991

22. Xia S, Liu X, Cao X, Xu S (2020) T-cell expression of Bruton's tyrosine kinase promotes autoreactive T-cell activation and exacerbates aplastic anemia. Cell Mol Immunol 17:1042-1052. https://doi.org/10.1038/s41423-019-0270-9

23. Bao Y, Zheng J, Han C et al (2012) Tyrosine kinase Btk is required for NK cell activation. J Biol Chem 287:2376923778. https://doi.org/10.1074/jbc.M112.372425

24. Wiestner A (2012) Emerging role of kinase-targeted strategies in chronic lymphocytic leukemia. Blood 120:4684-4691. https://doi.org/10.1182/blood-2012-05-423194

25. Reth M, Nielsen P (2014) Signaling circuits in early B-cell development. Adv Immunol 122:129-175. https://doi.org/10. 1016/B978-0-12-800267-4.00004-3

26. Dal Porto JM, Gauld SB, Merrell KT et al (2004) B cell antigen receptor signaling 101. Mol Immunol 41:599-613. https://doi. org/10.1016/j.molimm.2004.04.008

27. Takata M, Sabe H, Hata A et al (1994) Tyrosine kinases Lyn and Syk regulate B cell receptor-coupled $\mathrm{Ca} 2+$ mobilization through distinct pathways. EMBO J 13:1341-1349

28. Petro JB, Rahman SMJ, Ballard DW, Khan WN (2000) Bruton's Tyrosine kinase is required for activation of Iкb Kinase and nuclear factor $\kappa \mathrm{b}$ in response to B cell receptor engagement. J Exp Med 191:1745-1754. https://doi.org/10.1084/jem. 191.10.1745

29. Bajpai UD, Zhang K, Teutsch M et al (2000) Bruton's Tyrosine kinase links the B Cell receptor to nuclear factor $\mathrm{\kappa b}$ activation. J Exp Med 191:1735-1744. https://doi.org/10.1084/jem.191. 10.1735

30. Petro JB, Khan WN (2001) Phospholipase C- $\gamma 2$ couples Bruton's tyrosine kinase to the NF- $\mathrm{BB}$ signaling pathway in $\mathrm{B}$ lymphocytes. J Biol Chem 276:1715-1719. https://doi.org/10. 1074/jbc.M009137200

31. Dühren-von Minden M, Übelhart R, Schneider D et al (2012) Chronic lymphocytic leukaemia is driven by antigen-independent cell-autonomous signalling. Nature 489:309-312. https:// doi.org/10.1038/nature11309

32. de Rooij MFM, Kuil A, Geest CR et al (2012) The clinically active BTK inhibitor PCI-32765 targets B-cell receptor- and chemokine-controlled adhesion and migration in chronic lymphocytic leukemia. Blood 119:2590-2594. https://doi.org/10. 1182/blood-2011-11-390989

33. Aalipour A, Advani RH (2014) Bruton's tyrosine kinase inhibitors and their clinical potential in the treatment of B-cell malignancies: focus on ibrutinib. Ther Adv Hematol 5:121133. https://doi.org/10.1177/2040620714539906

34. Rawlings D, Saffran D, Tsukada S et al (1993) Mutation of unique region of Bruton's tyrosine kinase in immunodeficient XID mice. Science261 (80):358-361. https://doi.org/10.1126/ science. 8332901

35. Thomas J, Sideras P, Smith C, et al (1993) Colocalization of $\mathrm{X}$-linked agammaglobulinemia and X-linked immunodeficiency genes. Science 261(80):355-358. https://doi.org/10. 1126/science. 8332900
36. Tsukada S, Saffran DC, Rawlings DJ et al (1993) Deficient expression of a B cell cytoplasmic tyrosine kinase in human X-linked agammaglobulinemia. Cell 72:279-290. https://doi. org/10.1016/0092-8674(93)90667-F

37. Vetrie D, Vořechovský I, Sideras P et al (1993) The gene involved in X-linked agammaglobulinaemia is a member of the src family of protein-tyrosine kinases. Nature 361:226-233. https://doi.org/10.1038/361226a0

38. Satterthwaite AB (2018) Bruton's Tyrosine kinase, a component of B cell signaling pathways, has multiple roles in the pathogenesis of Lupus. Front Immunol 8:1986. https://doi.org/ 10.3389/fimmu.2017.01986

39. Gray P, Dunne A, Brikos C et al (2006) MyD88 adapter-like (Mal) is phosphorylated by Bruton's tyrosine kinase during TLR2 and TLR4 signal transduction. J Biol Chem 281:1048910495. https://doi.org/10.1074/jbc.M508892200

40. Liu X, Zhan Z, Li D et al (2011) Intracellular MHC class II molecules promote TLR-triggered innate immune responses by maintaining activation of the kinase Btk. Nat Immunol 12:416-424. https://doi.org/10.1038/ni.2015

41. Jefferies CA, Doyle S, Brunner C et al (2003) Bruton's tyrosine kinase is a Toll/interleukin-1 receptor domain-binding protein that participates in nuclear factor kappaB activation by Tolllike receptor 4. J Biol Chem 278:26258-26264. https://doi.org/ 10.1074/jbc.M301484200

42. Doyle SL, Jefferies CA, Feighery C, O'Neill LAJ (2007) Signaling by Toll-like receptors 8 and 9 requires Bruton's Tyrosine Kinase. J Biol Chem 282:36953-36960. https://doi.org/10. 1074/jbc.M707682200

43. Horwood NJ, Page TH, McDaid JP et al (2006) Bruton's Tyrosine Kinase is required for TLR2 and TLR4-induced TNF, but Not IL-6, production. J Immunol 176:3635-3641. https://doi. org/10.4049/jimmunol.176.6.3635

44. Spaargaren M, Beuling EA, Rurup ML et al (2003) The B cell antigen receptor controls integrin activity through Btk and PLCgamma2. J Exp Med 198:1539-1550. https://doi.org/10. 1084/jem.20011866

45. de Gorter DJJ, Beuling EA, Kersseboom R et al (2007) Bruton's tyrosine kinase and phospholipase Cgamma2 mediate chemokine-controlled B cell migration and homing. Immunity 26:93-104. https://doi.org/10.1016/j.immuni.2006.11.012

46. Halcomb KE, Contreras CM, Hinman RM et al (2007) Btk and phospholipase $\mathrm{C}$ gamma 2 can function independently during B cell development. Eur J Immunol 37:1033-1042. https://doi. org/10.1002/eji.200636451

47. Ritter SL, Hall RA (2009) Fine-tuning of GPCR activity by receptor-interacting proteins. Nat Rev Mol Cell Biol 10:819830. https://doi.org/10.1038/nrm2803

48. Park SH, Das BB, Casagrande F et al (2012) Structure of the chemokine receptor CXCR1 in phospholipid bilayers. Nature 491:779-783. https://doi.org/10.1038/nature11580

49. Lowry WE, Huang X-Y (2002) G Protein beta gamma subunits act on the catalytic domain to stimulate Bruton's agammaglobulinemia tyrosine kinase. J Biol Chem 277:1488-1492. https://doi.org/10.1074/jbc.M110390200

50. Langhans-Rajasekaran SA, Wan Y, Huang XY (1995) Activation of Tsk and Btk tyrosine kinases by $\mathrm{G}$ protein beta gamma subunits. Proc Natl Acad Sci 92:8601-8605. https://doi.org/ 10.1073/pnas.92.19.8601

51. Tsukada S, Simon MI, Witte ON, Katz A (1994) Binding of beta gamma subunits of heterotrimeric $G$ proteins to the PH domain of Bruton tyrosine kinase. Proc Natl Acad Sci 91:11256-11260. https://doi.org/10.1073/pnas.91.23.11256

52. Bence K, Ma W, Kozasa T, Huang XY (1997) Direct stimulation of Bruton's tyrosine kinase by G(q)-protein alpha-subunit. Nature 389:296-299. https://doi.org/10.1038/38520 
53. Bournazos S, Wang TT, Ravetch JV (2016) The role and function of Fc $\gamma$ receptors on myeloid cells. Microbiol Spectr 4 . https://doi.org/10.1128/microbiolspec.MCHD-0045-2016

54. Nimmerjahn F, Ravetch JV (2008) Fcgamma receptors as regulators of immune responses. Nat Rev Immunol 8:34-47. https:// doi.org/10.1038/nri2206

55. Bolland S, Pearse RN, Kurosaki T, Ravetch JV (1998) SHIP modulates immune receptor responses by regulating membrane association of Btk. Immunity 8:509-516. https://doi.org/10. 1016/S1074-7613(00)80555-5

56. Jongstra-Bilen J, Puig Cano A, Hasija M et al (2008) Dual functions of Bruton's Tyrosine Kinase and Tec Kinase during Fc $\gamma$ receptor-induced signaling and phagocytosis. J Immunol 181:288-298. https://doi.org/10.4049/jimmunol.181.1.288

57. Marron TU, Rohr K, Martinez-Gallo M et al (2010) TLR signaling and effector functions are intact in XLA neutrophils. Clin Immunol 137:74-80. https://doi.org/10.1016/j.clim.2010.06.011

58. Keaney J, Gasser J, Gillet G et al (2019) Inhibition of Bruton's Tyrosine Kinase modulates microglial phagocytosis: therapeutic implications for Alzheimer's disease. J Neuroimmune Pharmacol 14:448-461. https://doi.org/10.1007/s11481-019-09839-0

59. Burger JA (2014) Bruton's Tyrosine Kinase (BTK) inhibitors in clinical trials. Curr Hematol Malig Rep 9:44-49. https://doi.org/ 10.1007/s11899-013-0188-8

60. Tai Y-T, Chang BY, Kong S-Y et al (2012) Bruton tyrosine kinase inhibition is a novel therapeutic strategy targeting tumor in the bone marrow microenvironment in multiple myeloma. Blood 120:1877-1887. https://doi.org/10.1182/blood-2011-12-396853

61. Akinleye A, Chen Y, Mukhi N et al (2013) Ibrutinib and novel BTK inhibitors in clinical development. J Hematol Oncol 6:59. https://doi.org/10.1186/1756-8722-6-59

62. Herman SEM, Mustafa RZ, Gyamfi JA et al (2014) Ibrutinib inhibits BCR and NF- $\mathrm{KB}$ signaling and reduces tumor proliferation in tissue-resident cells of patients with CLL. Blood 123:3286-3295. https://doi.org/10.1182/blood-2014-02-548610

63. Zwolanek F, Riedelberger M, Stolz V et al (2014) The nonreceptor Tyrosine Kinase Tec controls assembly and activity of the noncanonical Caspase-8 inflammasome. PLoS Pathog 10:e1004525. https://doi.org/10.1371/journal.ppat.1004525

64. Massó-Vallés D, Jauset T, Serrano E et al (2015) Ibrutinib exerts potent antifibrotic and antitumor activities in mouse models of pancreatic adenocarcinoma. Cancer Res 75:1675-1681. https:// doi.org/10.1158/0008-5472.CAN-14-2852

65. Gao W, Wang M, Wang L et al (2014) Selective antitumor activity of Ibrutinib in EGFR-mutant non-small cell lung cancer cells. JNCI J Natl Cancer Inst 106. https://doi.org/10.1093/jnci/dju204

66. Soucek L, Buggy JJ, Kortlever R et al (2011) Modeling pharmacological inhibition of mast cell degranulation as a therapy for Insulinoma. Neoplasia 13:1093-IN43. https://doi.org/10.1593/ neo. 11980

67. Grabinski N, Ewald F (2014) Ibrutinib (ImbruvicaTM) potently inhibits ErbB receptor phosphorylation and cell viability of ErbB2-positive breast cancer cells. Invest New Drugs 32:10961104. https://doi.org/10.1007/s10637-014-0141-2

68. Honigberg LA, Smith AM, Sirisawad M et al (2010) The Bruton tyrosine kinase inhibitor PCI-32765 blocks B-cell activation and is efficacious in models of autoimmune disease and B-cell malignancy. Proc Natl Acad Sci 107:13075-13080. https://doi. org/10.1073/pnas.1004594107

69. Advani RH, Buggy JJ, Sharman JP et al (2013) Bruton tyrosine kinase inhibitor ibrutinib (PCI-32765) has significant activity in patients with relapsed/refractory B-cell malignancies. J Clin Oncol Off J Am Soc Clin Oncol 31:88-94. https://doi.org/10. 1200/JCO.2012.42.7906
70. Owen C, Berinstein NL, Christofides A, Sehn LH (2019) Review of Bruton tyrosine kinase inhibitors for the treatment of relapsed or refractory mantle cell lymphoma. Curr Oncol 26:e233-e240

71. Bye AP, Unsworth AJ, Desborough MJ et al (2017) Severe platelet dysfunction in NHL patients receiving ibrutinib is absent in patients receiving acalabrutinib. Blood Adv 1:2610-2623. https://doi.org/10.1182/bloodadvances.2017011999

72. Byrd JC, Harrington B, O'Brien S et al (2016) Acalabrutinib (ACP-196) in relapsed chronic lymphocytic leukemia. N Engl J Med 374:323-332. https://doi.org/10.1056/NEJMoa1509981

73. Wang M, Rule S, Zinzani PL et al (2018) Acalabrutinib in relapsed or refractory mantle cell lymphoma (ACE-LY-004): a single-arm, multicentre, phase 2 trial. Lancet (London, England) 391:659-667. https://doi.org/10.1016/S0140-6736(17)33108-2

74. ClinicalTrials.gov Study of Acalabrutinib (ACP-196) Versus Ibrutinib in previously treated subjects with high risk CLL

75. Melani C, Chiodoni C, Forni G, Colombo MP (2003) Myeloid cell expansion elicited by the progression of spontaneous mammary carcinomas in c-erbB-2 transgenic BALB/c mice suppresses immune reactivity. Blood 102:2138-2145. https://doi. org/10.1182/blood-2003-01-0190

76. Lechner MG, Liebertz DJ, Epstein AL (2010) Characterization of cytokine-induced myeloid-derived suppressor cells from normal human peripheral blood mononuclear cells. J Immunol 185:2273-2284. https://doi.org/10.4049/jimmunol.1000901

77. Gabrilovich DI, Nagaraj S (2009) Myeloid-derived suppressor cells as regulators of the immune system. Nat Rev Immunol 9:162-174. https://doi.org/10.1038/nri2506

78. Yu J, Wang Y, Yan F et al (2014) Noncanonical NF-кB activation mediates STAT3-stimulated IDO upregulation in myeloidderived suppressor cells in breast cancer. J Immunol 193:25742586. https://doi.org/10.4049/jimmunol.1400833

79. Flores RR, Clauson CL, Cho J et al (2017) Expansion of myeloid-derived suppressor cells with aging in the bone marrow of mice through a NF- $\mathrm{kB}$-dependent mechanism. Aging Cell 16:480-487. https://doi.org/10.1111/acel.12571

80. Stiff A, Trikha P, Mundy-Bosse B et al (2018) Nitric oxide production by myeloid-derived suppressor cells plays a role in impairing $\mathrm{Fc}$ receptor-mediated natural killer cell function. Clin Cancer Res 24:1891-1904. https://doi.org/10.1158/10780432.CCR-17-0691

81. Varikuti S, Singh B, Volpedo G et al (2020) Ibrutinib treatment inhibits breast cancer progression and metastasis by inducing conversion of myeloid-derived suppressor cells to dendritic cells. Br J Cancer 122:1005-1013. https://doi.org/10.1038/ s41416-020-0743-8

82. Bergenfelz C, Larsson A-M, von Stedingk K et al (2015) Systemic monocytic-MDSCs are generated from monocytes and correlate with disease progression in breast cancer patients. PLoS ONE 10:e0127028. https://doi.org/10.1371/journal.pone. 0127028

83. Fuertes MB, Kacha AK, Kline J et al (2011) Host type I IFN signals are required for antitumor CD8 $+\mathrm{T}$ cell responses through CD8\{alpha\}+ dendritic cells. J Exp Med 208:20052016. https://doi.org/10.1084/jem.20101159

84. Overman M, Javle M, Davis RE et al (2020) Randomized phase II study of the Bruton tyrosine kinase inhibitor acalabrutinib, alone or with pembrolizumab in patients with advanced pancreatic cancer. J Immunother Cancer 8. https://doi.org/10.1136/ jitc-2020-000587

85. Weide B, Martens A, Zelba H et al (2014) Myeloid-derived suppressor cells predict survival of patients with advanced melanoma: comparison with regulatory T cells and NY-ESO-1or melan-A-specific T cells. Clin cancer Res an Off J Am Assoc Cancer Res 20:1601-1609. https://doi.org/10.1158/1078-0432. CCR-13-2508 
86. Meyer C, Cagnon L, Costa-Nunes C-M et al (2013) Frequencies of circulating MDSC correlate with clinical outcome of melanoma patients treated with ipilimumab. Cancer Immunol Immunother 63. https://doi.org/10.1007/s00262-013-1508-5

87. Benner B, Quiroga DM, Good L et al (2020) A pilot study of Bruton's tyrosine kinase inhibitor ibrutinib alone and in combination with PD-1 inhibitor nivolumab in patients with metastatic solid tumors. J Clin Oncol 38:3111-3111. https:// doi.org/10.1200/JCO.2020.38.15_suppl.3111

88. Chen Y, Song Y, Du W et al (2019) Tumor-associated macrophages: an accomplice in solid tumor progression. J Biomed Sci 26:78. https://doi.org/10.1186/s12929-019-0568-Z

89. Yang L, Zhang Y (2017) Tumor-associated macrophages: from basic research to clinical application. J Hematol Oncol 10:58. https://doi.org/10.1186/s13045-017-0430-2

90. Sousa S, Brion R, Lintunen M et al (2015) Human breast cancer cells educate macrophages toward the M2 activation status. Breast Cancer Res 17:101. https://doi.org/10.1186/ s13058-015-0621-0

91. Dehne N, Mora J, Namgaladze D et al (2017) Cancer cell and macrophage cross-talk in the tumor microenvironment. Curr Opin Pharmacol 35:12-19. https://doi.org/10.1016/j.coph.2017. 04.007

92. Ping L, Ding N, Shi Y et al (2017) The Bruton's tyrosine kinase inhibitor ibrutinib exerts immunomodulatory effects through regulation of tumor-infiltrating macrophages. Oncotarget 8:3921839229. https://doi.org/10.18632/oncotarget.16836

93. Gunderson AJ, Kaneda MM, Tsujikawa T et al (2016) Bruton tyrosine kinase-dependent immune cell cross-talk drives pancreas cancer. Cancer Discov 6:270-285. https://doi.org/10.1158/ 2159-8290.CD-15-0827

94. Shibuya KC, Goel VK, Xiong W et al (2014) Pancreatic ductal adenocarcinoma contains an effector and regulatory immune cell infiltrate that is altered by multimodal neoadjuvant treatment. PLoS ONE 9:e96565. https://doi.org/10.1371/journal.pone. 0096565

95. Qiu Q, Li C, Song Y et al (2019) Targeted delivery of ibrutinib to tumor-associated macrophages by sialic acid-stearic acid conjugate modified nanocomplexes for cancer immunotherapy. Acta Biomater 92:184-195. https://doi.org/10.1016/j.actbio.2019.05. 030

96. Lee J, Wan J, Lee L et al (2017) Study of the NLRP3 inflammasome component genes and downstream cytokines in patients with type 2 diabetes mellitus with carotid atherosclerosis. Lipids Health Dis 16:217. https://doi.org/10.1186/s12944-017-0595-2

97. Chang BY, Huang MM, Francesco M et al (2011) The Bruton tyrosine kinase inhibitor PCI-32765 ameliorates autoimmune arthritis by inhibition of multiple effector cells. Arthritis Res Ther 13:R115. https://doi.org/10.1186/ar3400

98. Eltzschig HK, Eckle T (2011) Ischemia and reperfusion-from mechanism to translation. Nat Med 17:1391-1401. https://doi. org/10.1038/nm.2507

99. Iadecola C, Anrather J (2011) The immunology of stroke: from mechanisms to translation. Nat Med 17:796-808. https://doi.org/ 10.1038/nm.2399

100. Ye B, Zhou C, Guo H, Zheng M (2019) Effects of BTK signalling in pathogenic microorganism infections. J Cell Mol Med 23:6522-6529. https://doi.org/10.1111/jcmm.14548

101. Brunner C, Müller B, Wirth T (2005) Bruton's Tyrosine Kinase is involved in innate and adaptive immunity. Histol Histopathol 20:945-955. https://doi.org/10.14670/HH-20.945

102. de Porto AP, Liu Z, de Beer R et al (2019) Btk inhibitor ibrutinib reduces inflammatory myeloid cell responses in the lung during murine pneumococcal pneumonia. Mol Med 25:3. https://doi. org/10.1186/s10020-018-0069-7
103. Olsson S, Sundler R (2006) Different roles for non-receptor tyrosine kinases in arachidonate release induced by zymosan and Staphylococcus aureus in macrophages. J Inflamm (Lond) 3:8. https://doi.org/10.1186/1476-9255-3-8

104. Köprülü AD, Kastner R, Wienerroither S et al (2013) The tyrosine kinase Btk regulates the macrophage response to Listeria monocytogenes infection. PLoS ONE 8:e60476. https://doi.org/ 10.1371/journal.pone.0060476

105. Mukhopadhyay S, Sahoo PK, George A et al (1999) Delayed clearance of filarial infection and enhanced Th1 immunity due to modulation of macrophage APC functions in xid mice. J Immunol 163:875-883

106. Colado A, Genoula M, Cougoule C et al (2018) Effect of the BTK inhibitor ibrutinib on macrophage- and $\gamma \delta$ T cell-mediated response against Mycobacterium tuberculosis. Blood Cancer J 8:100. https://doi.org/10.1038/s41408-018-0136-x

107. Strijbis K, Tafesse FG, Fairn GD et al (2013) Bruton's Tyrosine Kinase (BTK) and Vav1 contribute to Dectin1-dependent phagocytosis of Candida albicans in macrophages. PLoS Pathog 9:e1003446. https://doi.org/10.1371/journal.ppat.1003446

108. Shahan TA, Sorenson WG, Simpson J et al (2000) Tyrosine kinase activation in response to fungal spores is primarily dependent on endogenous reactive oxygen production in macrophages. J Biol Chem 275:10175-10181. https://doi.org/10. 1074/jbc.275.14.10175

109. Szymczak WA, Davis MJ, Lundy SK et al (2013) X-linked immunodeficient mice exhibit enhanced susceptibility to Cryptococcus neoformans Infection. MBio 4. https://doi.org/10.1128/mBio. 00265-13

110. Herbst S, Shah A, Mazon Moya M et al (2015) Phagocytosisdependent activation of a TLR9-BTK-calcineurin-NFAT pathway co-ordinates innate immunity to Aspergillus fumigatus. EMBO Mol Med 7:240-258. https://doi.org/10.15252/emmm. 201404556

111. Bercusson A, Colley T, Shah A et al (2018) Ibrutinib blocks Btkdependent NF-KB and NFAT responses in human macrophages during Aspergillus fumigatus phagocytosis. Blood 132:19851988. https://doi.org/10.1182/blood-2017-12-823393

112. Colado A, Marín Franco JL, Elías EE et al (2020) Second generation BTK inhibitors impair the anti-fungal response of macrophages and neutrophils. Am J Hematol 95:E174-E178

113. Shinohara M, Chang BY, Buggy JJ et al (2014) The orally available Btk inhibitor ibrutinib (PCI-32765) protects against osteoclast-mediated bone loss. Bone 60:8-15. https://doi.org/10. 1016/j.bone.2013.11.025

114. Hartkamp LM, Fine JS, van Es IE et al (2015) Btk inhibition suppresses agonist-induced human macrophage activation and inflammatory gene expression in RA synovial tissue explants. Ann Rheum Dis 74:1603-1611. https://doi.org/10.1136/annrh eumdis-2013-204143

115. Zhou P, Ma B, Xu S et al (2014) Knockdown of Burton's tyrosine kinase confers potent protection against sepsis-induced acute lung injury. Cell Biochem Biophys 70:1265-1275. https://doi. org/10.1007/s12013-014-0050-1

116. Bender AT, Pereira A, Fu K et al (2016) Btk inhibition treats TLR7/IFN driven murine lupus. Clin Immunol 164:65-77. https://doi.org/10.1016/j.clim.2016.01.012

117. Mangla A, Khare A, Vineeth V et al (2004) Pleiotropic consequences of Bruton tyrosine kinase deficiency in myeloid lineages lead to poor inflammatory responses. Blood 104:1191-1197. https://doi.org/10.1182/blood-2004-01-0207

118. Mao L, Kitani A, Hiejima E et al (2020) Bruton tyrosine kinase deficiency augments NLRP3 inflammasome activation and causes IL-1 $\beta$-mediated colitis. J Clin Invest 130:1793-1807. https://doi.org/10.1172/JCI128322 
119. Lee K-G, Xu S, Kang Z-H et al (2012) Bruton's tyrosine kinase phosphorylates Toll-like receptor 3 to initiate antiviral response. Proc Natl Acad Sci U S A 109:5791-5796. https://doi.org/10. 1073/pnas.1119238109

120. Florence JM, Krupa A, Booshehri LM et al (2018) Inhibiting Bruton's tyrosine kinase rescues mice from lethal influenzainduced acute lung injury. Am J Physiol Cell Mol Physiol 315:L52-L58. https://doi.org/10.1152/ajplung.00047.2018

121. Chong EA, Roeker LE, Shadman M et al (2020) BTK inhibitors in cancer patients with COVID19: "The winner will be the one who controls that chaos"; (Napoleon Bonaparte). Clin Cancer Res clincanres.1427.2020. https://doi.org/10.1158/1078-0432. CCR-20-1427

122. Roschewski M, Lionakis MS, Sharman JP et al (2020) Inhibition of Bruton tyrosine kinase in patients with severe COVID-19. Sci Immunol 5:eabd0110. https://doi.org/10.1126/sciimmunol.abd01 10

123. Treon SP, Castillo JJ, Skarbnik AP et al (2020) The BTK inhibitor ibrutinib may protect against pulmonary injury in
COVID-19-infected patients. Blood 135:1912-1915. https://doi. org/10.1182/blood.2020006288

124. Wang D, Hu B, Hu C et al (2020) Clinical characteristics of 138 hospitalized patients with 2019 novel Coronavirus-infected Pneumonia in Wuhan. China JAMA 323:1061. https://doi.org/ 10.1001/jama.2020.1585

125. Bhatraju PK, Ghassemieh BJ, Nichols M et al (2020) Covid-19 in critically Ill patients in the Seattle region-case series. N Engl J Med NEJMoa2004500. https://doi.org/10.1056/NEJMoa2004 500

Publisher's Note Springer Nature remains neutral with regard to jurisdictional claims in published maps and institutional affiliations. 